\title{
A NEW GLOBAL CATALOGUE OF LUNAR CRATERS ( $\geq 1$ KM) WITH 3D INFORMATION AND PRELIMINARY RESULTS OF GLOBAL ANALYSIS
}

\author{
Yiran Wang, Bo Wu* \\ Department of Land Surveying and Geo-Informatics, The Hong Kong Polytechnic University, Hung Hom, Kowloon, Hong Kong \\ - (yiran.wang@ connect.polyu.hk; bo.wu@ polyu.edu.hk)
}

Commission III, ICWG III/II

KEY WORDS: Moon, Crater, Catalogue, DEM, Crater Distribution, Crater Morphology

\begin{abstract}
:
Impact craters are the predominant geomorphological features on the lunar surface. They can be studied to infer the ages of the lunar surfaces, the generation processes of the geological units, and the sequences of its geological events. Lunar crater-related research is dependent on crater records, which are usually stored in the form of crater catalogues. In the past, various efforts have been dedicated to generating global lunar crater catalogues. All published global catalogues, however, either contain only relatively large craters or lack 3D morphological information. This paper first presents approaches for automatic crater detection and the extraction of crater morphological information. The approaches have been performed on the lunar global datasets, e.g., digital elevation models (DEMs) and images, resulting in a global catalogue of lunar craters. To guarantee the reliability of the crater detection results, intensive manualchecking processes have been performed to improve the correctness and completeness of the catalogue. The generated global catalogue contains entries on 1.31 million lunar craters. It extends the existing global catalogues to craters with diameters $\geq 1 \mathrm{~km}$ and enriched with 3D morphological information of craters. Global analyses of craters were conducted based on the newly generated catalogue, including the analysis of crater density and depth-to-diameter ratio. We re-examined the previously observed distributions and patterns to show its fidelity and further explored other global relationships, which have not been discovered in previous research. The results updated the clues on impact cratering process and terrain differences. The developed global catalogue of lunar craters can be utilised for different applications by the research community, and the relevant research will help to enrich the literature and facilitate the advancement of crater-related planetary science.
\end{abstract}

\section{INTRODUCTION}

Craters are dominant geomorphological features on the lunar surface. Lunar crater catalogues provide the basis for investigating crater populations, distributions and their morphometric parameters, which are valuable information on impact cratering processes as well as the physical and geological processes of planetary bodies. The applications of lunar crater catalogues range from dating lunar surface age (Neukum et al., 2001), investigating the formation processes of geological units and the sequences of geological events (Head et al., 2010), and selecting the landing site for lunar exploration missions (De Rosa et al., 2012; Wu et al., 2018; Wu et al., 2014).

Lunar crater-related research is dependent on crater records, which are usually stored in the form of crater catalogues. Extensive efforts have been made to generate global or local catalogues of lunar impact craters. However, due to data limitations and time-consuming human-performed processes, most catalogues contain only large craters. The first cataloguing efforts of an all-encompassing lunar global crater catalogue in a specific diameter range were made by Head et al. (2010). Their catalogue contained entries on 5,185 craters with diameters $>20$ $\mathrm{km}$, and these were manually digitised based on the earlier digital elevation models (DEMs) produced from the Lunar Orbiter Laser Altimeter (LOLA; Smith et al., 2010) onboard NASA's Lunar Reconnaissance Orbiter (LRO). Later works extended this catalogue to contain craters larger than $10 \mathrm{~km}$ (Luo et al., 2013), $8 \mathrm{~km}$ (Salamunićcar et al., 2014; Salamunićcar et al., 2012), $5 \mathrm{~km}$ (Barlow, 2017) and $3 \mathrm{~km}$ (Krüger et al., 2018) in diameter.
Among these crater catalogues, only the one by Krüger et al. (2018) provided 3D morphological information of craters. However, research on smaller craters is essential, as it can, for example, increase the reliability of age analysis and reveal the resurfacing process with more details. The recently published lunar crater catalogue by Robbins (2018) includes craters with a minimum diameter of $1-2 \mathrm{~km}$. It is the most up-to-date lunar crater catalogue in terms of sizes and number of craters covered, but it does not include 3D morphological information of craters. Morphological data such as crater depth, bottom width and rim height are crucial to facilitate a variety of investigations of planetary processes, terrain properties, cratering mechanisms, and geology. Quantification of crater morphologies is therefore essential for understanding the abovementioned aspects. Prior extractions of 3D morphological information on craters have focused on small, isolated lunar regions or a small number of specific types of craters (Krüger et al., 2018; Pike, 1977a; Pike, 1977b; Wünnemann and Ivanov, 2003). Global studies of crater morphologies, including those of small craters, have not been conducted due to the lack of a complete global database and the heavy workload required. Thus, it is highly desirable for a global crater catalogue covering a broad range of sizes and containing 3D morphological information to be created.

The higher-resolution remote sensing datasets collected by recent lunar missions enable the investigations of craters with a relatively smaller size and provide more accurate and detailed 3D information. In this paper, an automatic crater detection approach and an approach for 3D morphological information extraction

\footnotetext{
* Corresponding author
} 
have been developed. Based on the developed approaches, a new global catalogue of lunar craters has been generated, which contains entries for 1.31 million craters $\geq 1 \mathrm{~km}$ in diameter and with detailed 3D morphological information. This paper also presents the preliminary results for a global analysis of lunar craters based on the new catalogue.

\section{APPROACHES FOR CRATER DETECTION AND MORPHOLOGICAL INFORMATION EXTRACTION}

\subsection{Machine Learning for Crater Detection Based on Lunar DEMs}

The generation of a lunar crater catalogue requires more automated methods of crater detection, as it involves a large number of datasets. A machine-learning approach for automatic crater detection based on DEMs has been developed based on our previous works (Wang et al., 2018; Wang and Wu, 2020). First, a feature descriptor based on the histogram of oriented gradient (HOG; Dalal and Triggs, 2005) was designed for describing the elevation changes of crater areas and extracting features for machine learning. Figure 1 shows examples of the extracted HOG features for a crater DEM and a non-crater DEM. Then, a support vector machine (SVM) classifier was trained and optimised using the HOG features from positive samples (crater regions) and negative samples (non-crater regions). The resulting trained classifier was then used to detect craters on DEMs and was able to return both the centre coordinate and the diameter of each crater. The classifier was trained using a collection of positive and negative samples and validated independently using data at other different sites. The tests confirm the effectiveness and high efficiency of the proposed approach, which allows the crater detection to be conducted globally.

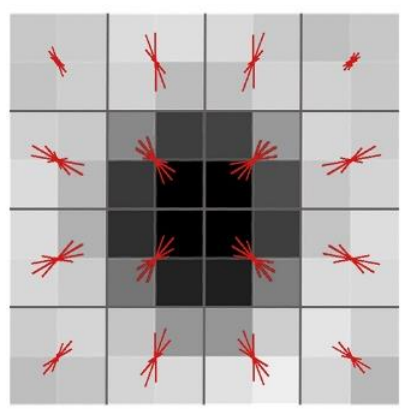

Crater DEM

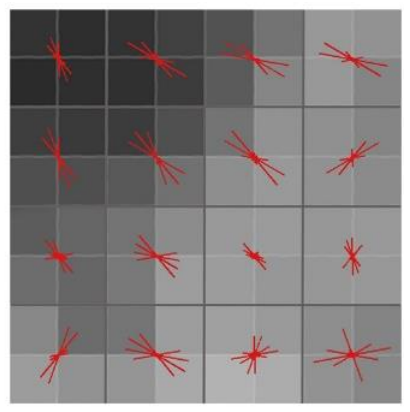

Non-Crater DEM
Figure 1. Illustration of the extracted HOG features for a crater DEM and a non-crater DEM.

\subsection{D Morphological Information Extraction}

In addition to extracting the geographic coordinates of the crater centres and diameters of craters by the machine learning approach, an effective approach for extraction of 3D morphological information of craters (including crater depth, wall slope, bottom width, rim height, central peak height and width) has been developed, based on the 3D information of the DEMs. First, two profiles are derived from each crater, namely a vertical profile, and a horizontal profile. A two-term Gaussian fitting is then applied for each profile (as illustrated in Figure 2) to remove possible noise (e.g., local elevation changes). Second, the morphological parameters are calculated from the well-fitted profiles. The two-term Gaussian fitting may fail due to the irregular shape of the profile when craters are overlapped or highly degraded; in this case, the profile is excluded from the calculation. Finally, the morphological parameters of the craters are determined by adopting the average or maximum of the parameters calculated from different profiles. If both extracted profiles fail in the Gaussian fitting, the crater is regarded as a wrong detection and will be removed from the crater catalogue.

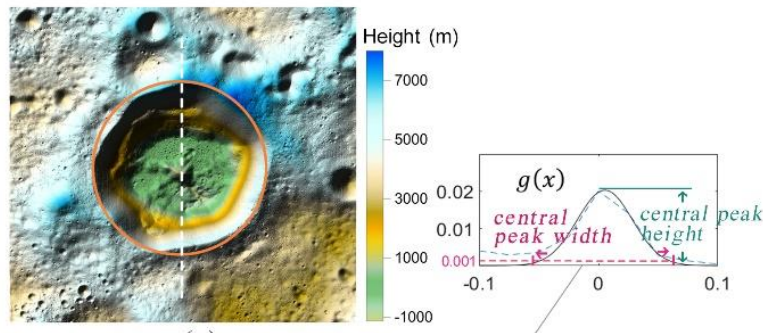

(a)

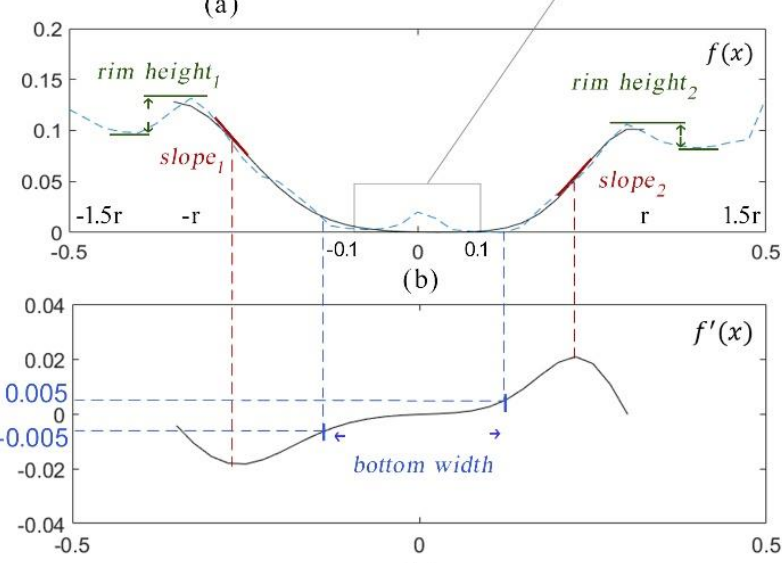

(c)

Figure 2. Extraction of 3D morphological information of craters based on Gaussian fitting. (a) The circle shows the boundary of the detected crater, and the dashed line illustrates where the crater profile is extracted. (b) The light-blue dashed line displays the extracted profile, and the black line shows the fitted two-term Gaussian function. For the sake of fitting, the horizontal distance (x-axis) is normalised, and the elevation (y-axis) is enlarged accordingly. The enlarged part on the left shows the central peak fitted by the one-term Gaussian function. (c) The black line is the first-order derivative of the fitted Gaussian function, used for the calculation of morphological information.

\section{THE NEW GLOBAL CRATER CATALOGUE}

\subsection{Generation of the Global Crater Catalogue}

The approaches mentioned above for crater detection and morphological information extraction were applied on DEMs covering the entire lunar surfaces, resulted in initial global crater detection results $(\geq 1 \mathrm{~km}$ in diameter) with $3 \mathrm{D}$ morphological information. For the regions in latitudes of $\left[-60^{\circ}, 60^{\circ}\right]$, the LOLA and SELENE Terrain Camera (TC; Kato et al., 2008) merged DEM (SLDEM; Barker et al., 2016) was used. The SLDEM has a spatial resolution of 512 pixels per degree (about $60 \mathrm{~m}$ at the equator) and a vertical accuracy of about 3 to $4 \mathrm{~m}$. For the regions in latitudes of $\left[-90^{\circ},-60^{\circ}\right]$ and $\left[60^{\circ}, 90^{\circ}\right]$, the LOLA DEM (LDEM; Smith et al., 2010) was used. The LDEM has the best resolution of 1,024 pixels per degree and a relatively better resolution on polar regions due to the principle of laser altimetry. The SELENE TC images with a spatial resolution of $10 \mathrm{~m} / \mathrm{pixel}$ and the Digital Orthophoto Map (DOM) from the Chang'E-2 images (Li et al., 2018) with a spatial resolution of $7 \mathrm{~m} /$ pixel were employed in the manual checking process. The SLDEM, LDEM, SELENE TC images and Chang'E-2 images were manually coregistered with the LDEM as a base map to remove the geometric inconsistencies among them before their use for crater detection. 
In the above process, the Mercator projection was used for regions between latitudes $\pm 75^{\circ}$, with a central meridian at $0^{\circ}$; while those poleward of $75^{\circ}$ were processed in the polar stereographic projection, with a central meridian set for both at $0^{\circ}$. The final catalogue was projected in Moon 2000 coordinate system and the adopted spherical radius used is $1737.4 \mathrm{~km}$. The coordinates of the craters were recorded in latitude and longitude. The distortions of morphological parameters caused by Mercator projection have been removed.

To guarantee the reliability of the global crater detection, tremendous efforts have been paid to check the correctness and completeness of the detected craters by a group of independent operators. A grid with a cell size of $1 \mathrm{~km} \mathrm{x} 1 \mathrm{~km}$ was overlaid on the TC and Chang'E-2 images to assist the manual checking of the detected craters and digitization of possible craters missed by the automatic algorithm. Besides, previous crater catalogues (Head et al. 2010; Povilaitis et al. 2018; Robbins 2018) were overlaid on our results as references. The inconsistences between our catalogue and the previous ones were given extra attention. The checking processes were conducted by several independent operators, and the divergences were further discussed.
All detected craters, regardless of degradation state, were included in the catalogue as long as they could be recognised from DEMs with measurable rims. As such, the catalogue includes newly formed craters, as well as those degraded or partially buried. Highly eroded 'ghost' craters were not included in the catalogue.

The final generated crater catalogue contains entries on 1.31 million craters with diameters $\geq 1 \mathrm{~km}$. Each entry comprises the geographic coordinates of the centre, diameter, and the 3D morphological information for each crater, i.e., crater depth, wall slope, bottom width, rim height, central peak height and central peak width. An overview of the global crater catalogue is shown in Figure 3. The catalogue is provided in both text and shapefile (for direct use in ArcGIS) formats. The shapefile version is divided into 24 bins separated by $15^{\circ}$ interval in longitude, and detailed crater information is recorded in the attribute tables.

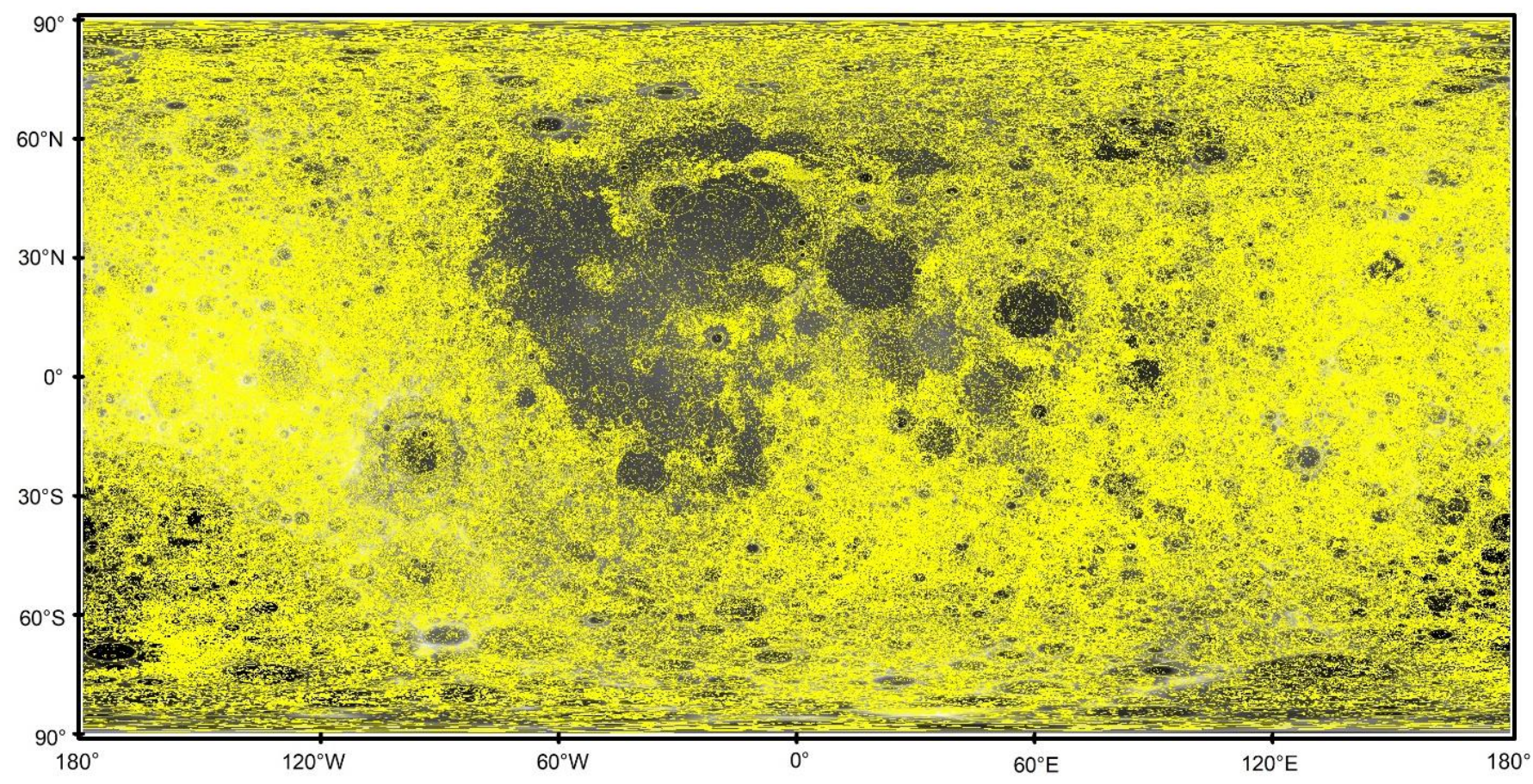

Figure 3. The new global catalogue of lunar craters $(\geq 1 \mathrm{~km})$ overlaid on the SLDEM and LDEM.

\subsection{Comparison with Previous Crater Catalogues}

The developed global catalogue of lunar craters was compared with the three most representative lunar crater catalogues, namely those by Head et al. (2010), Povilaitis et al. (2018) and Robbins (2018). Overall, for craters with diameters $\geq 5 \mathrm{~km}$, our crater catalogue shows good agreement with the catalogue by Robbins (2018) but contains more entries than those in the catalogues by Povilaitis et al. (2018) and Head et al. (2010). In the diameter range of $1-5 \mathrm{~km}$, the number of craters in our catalogue is more than those in Robbins (2018).

The inconsistencies between our catalogue and the catalogues by Head et al. (2010), Povilaitis et al. (2018) and Robbins (2018) are considered due to the following reasons: (1) the crater identification for each catalogue may have errors and uncertainties; (2) the newly released data with better resolution and quality enabled detection of new and small craters that might have been easily missed in previous catalogues; and (3) different crater catalogue might have different criteria for crater inclusion, which may also influence the consistency among crater catalogues. For example, some craters buried by mare but still observable on the surface were not included in previous catalogues (e.g., Povilaitis et al. 2018), but were identified and included in our catalogue. Besides, the catalogue by Robbins (2018) contains entries for craters $>1-2 \mathrm{~km}$ while our catalogue contains entries for craters $\geq 1 \mathrm{~km}$; thus, the catalogue by Robbins (2018) has fewer craters in the diameter range of 1-2 km. 


\section{PRELIMINARY RESULTS OF GLOBAL ANALYSIS}

\subsection{Global Crater Density}

The crater density is the number of craters per unit area. For craters with a diameter $>20 \mathrm{~km}$, a moving window of $500 \mathrm{~km}$ was adopted to align the results with those of Head (2010). For craters $\leq 20 \mathrm{~km}$, a smaller moving window of $100 \mathrm{~km}$ was used to preserve more details. To achieve a fair comparison with previous works in light of different sizes of moving windows, the normalised crater density was adopted, which calculated the number of craters with diameter $\mathrm{D} \geq \mathrm{d}$ per $10^{6}$. This normalised crater density has commonly been adopted by the research community (Fassett et al., 2012; Head et al., 2010; Robbins, 2018). The density maps in Figure 4 are presented in normalised crater density.
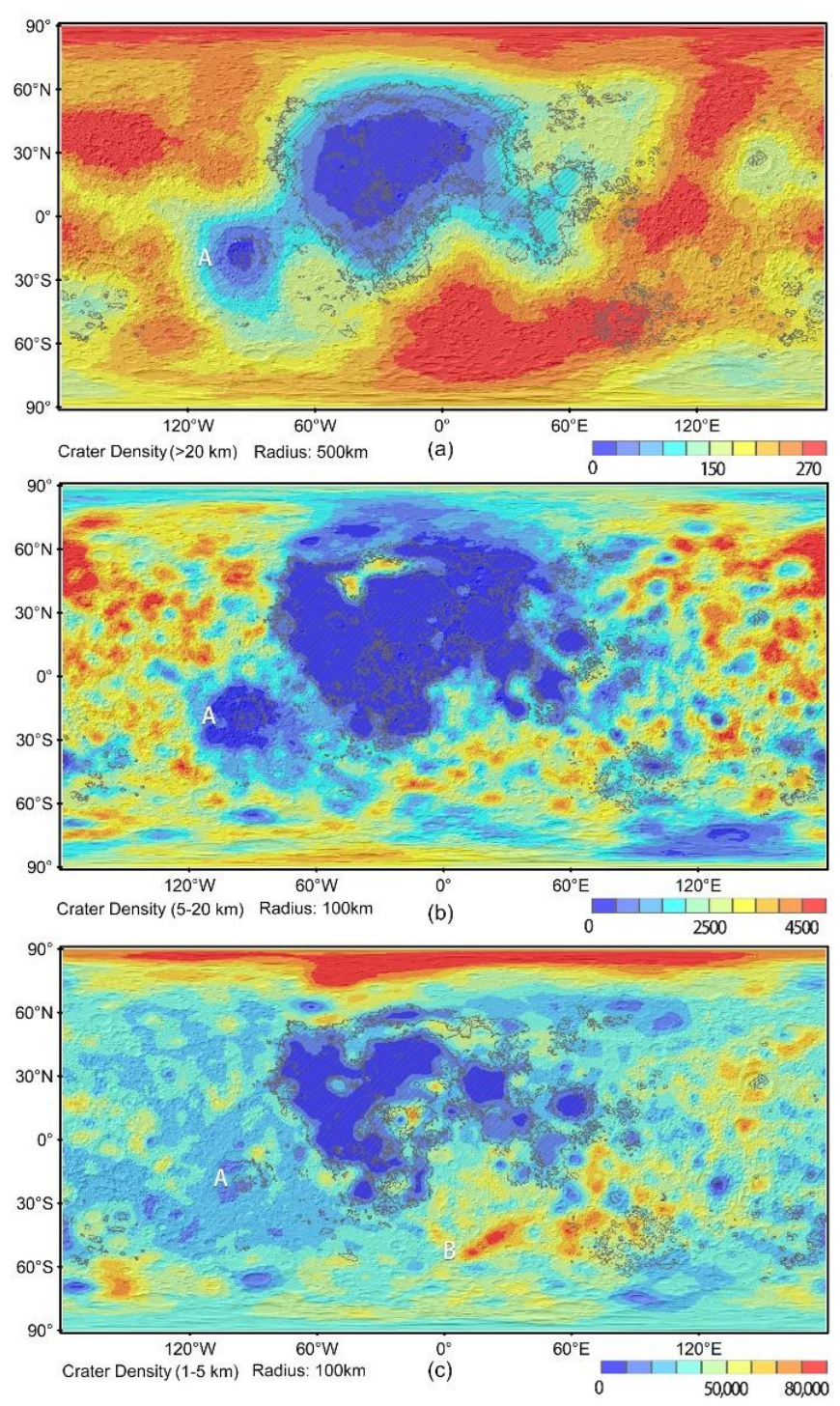

Figure 4. Global normalized crater density maps for (a) $>20 \mathrm{~km}$, (b) 5-20 km and (c) $1-5 \mathrm{~km}$. The lunar mare regions are marked by grey slashes.

The density maps of craters $>20 \mathrm{~km}$ and 5-20 km (Figure 4) are in good agreement with previous results by Head et al. (2010) and Robbins (2018). The crater density map of $1-5 \mathrm{~km}$, however, shows more detailed trends that have not been reported before, which include: (1) the boundaries of high density and low density show a high similarity to the boundaries between lunar mare and the highlands; (2) the Orientale basin (marked by 'A'), which shows an apparent low density for large craters, has no noticeable density difference for $1-5 \mathrm{~km}$ diameter craters; and (3) highdensity areas of $1-5 \mathrm{~km}$ craters are located at the north pole and a local region $\left(5-80^{\circ} \mathrm{E}, 30-50^{\circ} \mathrm{S}\right.$; marked by ' $\mathrm{B}$ ' in Figure $\left.4 \mathrm{c}\right)$.

\subsection{Crater Depth-to-Diameter Ratio}

The 3D morphological information of craters available in our catalogue enables the study of crater depth-to-diameter relationships. To evaluate the spatial association between the depth-to-diameter ratios and locations, the average depth-todiameter ratios of craters in different diameter ranges were measured in a moving window with a $100 \mathrm{~km}$ radius, as shown in Figure 5. Overall, the spatial distribution of the average depthto-diameter ratios shows apparent discrepancies between lunar highland and mare. The majority of lunar mare regions have an average depth-to-diameter ratio of lager than 0.1 , while in comparison, the highland regions show a significantly lower value. The morphological differences of craters on mare and highland have been noticed before, and the reason may be the differences in strength of the target material (e.g., Senft and Stewart, 2007). There was an agreement that complex craters on highland terrains are deeper than those on the lunar mare (Kalynn et al., 2013; Pike, 1981; Pike, 1980). However, our results show the opposite trend that the craters on the lunar mare are relative deeper than those on the highlands. The possible reason for this disagreement might be that our average depth-to-diameter map was most contributed by small craters of $1-2 \mathrm{~km}$ diameter. In comparison, previous research focused on complex craters that larger than $10 \mathrm{~km}$ or $20 \mathrm{~km}$. The craters in different sizes show diverse morphology on the lunar mare and highlands.

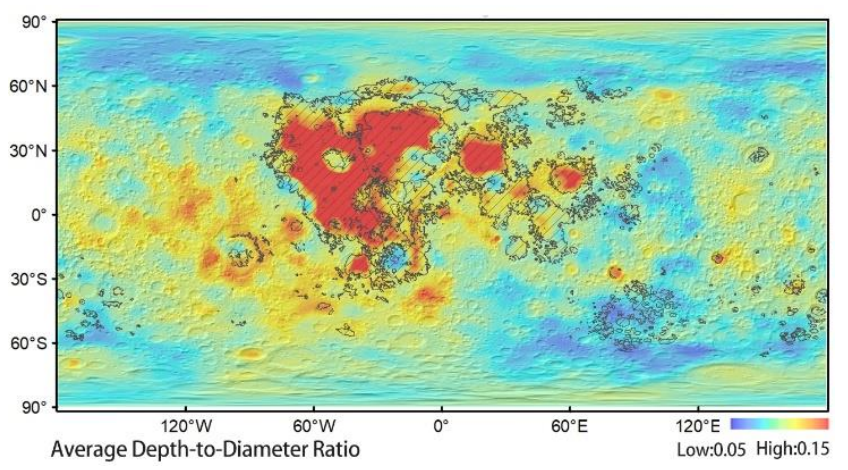

Figure 5. The average depth-to-diameter ratios calculated in a moving window of $100 \mathrm{~km}$ radius. The regions of the lunar mare are marked by grey slashes.

\section{CONCLUSIONS AND DISCUSSION}

This paper presents a new global catalogue of lunar craters. It extends the existing global catalogues to craters with diameters $\geq$ $1 \mathrm{~km}$. Furthermore, with the availability of 3D information extracted from the DEMs, the crater catalogue is enriched by $3 \mathrm{D}$ morphological information of craters. This new catalogue is more complete than any previously published catalogue and will be useful for researchers in lunar geology and other related fields.

Based on the new global catalogue of lunar craters, global crater analyses were conducted, including the analysis of crater density, and crater depth-to-diameter ratio. Preliminary results of the global analysis are summarised as follows: 
1. The global crater density maps of large craters $(>20 \mathrm{~km}$ in diameter) were in good agreement with previous results. The $1-5 \mathrm{~km}$ diameter crater density map had a better spatial resolution. It showed that the boundaries of high-density and low-density areas have a high similarity to the boundaries between lunar mare and the highlands.

2. The analysis of depth-to-diameter ratios revealed that small craters (about 1-2 km) on the lunar mare have larger average depth-to-diameter ratios than that on the highlands. This was in contrast to the previous conclusion that craters on the highlands were deeper than those on the mare.

Our future efforts will be the investigation of crater distribution and morphological characteristics in other regions, such as the Imbrium Basin, the Nectaris Basin and polar regions. The developed lunar crater catalogue can be utilised for different purposes by the research community, and the derived research will help to enrich the literature and facilitate the advancement of crater-related planetary science.

\section{ACKNOWLEDGEMENTS}

The work described in this paper was funded by a grant from the Research Grants Council of Hong Kong (Research Impact Fund - Project No: R5043-19) and a grant from the National Natural Science Foundation of China (Project No: 41671426). The authors also would like to thank all those who worked on the archive of the DEM and other datasets to make them publicly available.

\section{REFERENCES}

Barker, M., Mazarico, E., Neumann, G., Zuber, M., Haruyama, J., Smith, D., 2016. A new lunar digital elevation model from the Lunar Orbiter Laser Altimeter and SELENE Terrain Camera. Icarus 273, 346-355.

Barlow, N., 2017. Status Report on Crater Databases for Mercury, the Moon, Mars, and Ganymede, Third Planetary Data Workshop and The Planetary Geologic Mappers Annual Meeting.

Dalal, N., Triggs, B., 2005. Histograms of oriented gradients for human detection, international Conference on computer vision \& Pattern Recognition (CVPR'05). IEEE Computer Society pp. 886--893.

De Rosa, D., Bussey, B., Cahill, J.T., Lutz, T., Crawford, I.A., Hackwill, T., van Gasselt, S., Neukum, G., Witte, L., McGovern, A., 2012. Characterisation of potential landing sites for the European Space Agency's Lunar Lander project. Planetary and Space Science 74, 224-246.

Gault, D.E., 1970. Saturation and equilibrium conditions for impact cratering on the lunar surface: Criteria and implications. Radio Science 5, 273-291.

Group, C.A.T.W., 1979. Standard techniques for presentation and analysis of crater size-frequency data. Icarus 37, 467-474.

Head, J.W., Fassett, C.I., Kadish, S.J., Smith, D.E., Zuber, M.T., Neumann, G.A., Mazarico, E., 2010. Global distribution of large lunar craters: Implications for resurfacing and impactor populations. Science 329, 1504-1507.
Kalynn, J., Johnson, C.L., Osinski, G.R., Barnouin, O., 2013. Topographic characterization of lunar complex craters. Geophysical Research Letters 40, 38-42.

Kato, M., Sasaki, S., Tanaka, K., Iijima, Y., Takizawa, Y., 2008. The Japanese lunar mission SELENE: Science goals and present status. Advances in Space Research 42, 294-300.

Krüger, T., Hergarten, S., Kenkmann, T., 2018. Deriving Morphometric Parameters and the Simple-to-Complex Transition Diameter From a High-Resolution, Global Database of Fresh Lunar Impact Craters $(\mathrm{D} \geq \sim 3 \mathrm{~km})$. Journal of Geophysical Research: Planets 123(10), 2667-2690.

Li, C., Liu, J., Ren, X., Yan, W., Zuo, W., Mu, L., Zhang, H., Su, Y., Wen, W., Tan, X., 2018. Lunar Global High-precision Terrain Reconstruction Based on Chang'e-2 Stereo Images.

Luo, L., Mu, L., Wang, X., Li, C., Ji, W., Zhao, J., Cai, H., 2013. Global detection of large lunar craters based on the CE-1 digital elevation model. Frontiers of Earth Science 7, 456-464.

Neukum, G., Ivanov, B.A., Hartmann, W.K., 2001. Cratering records in the inner solar system in relation to the lunar reference system, Chronology and evolution of Mars. Springer, pp. 55-86.

Pike, R., 1977a. Apparent depth/apparent diameter relation for lunar craters, Lunar and planetary science conference proceedings, pp. 3427-3436.

Pike, R., 1981. Target-dependence of crater depth on the Moon, Lunar and Planetary Science Conference, pp. 845-847.

Pike, R.J., 1977b. Size-dependence in the shape of fresh impact craters on the Moon. Impact and explosion cratering: Planetary and terrestrial implications, pp. 489-509.

Pike, R.J., 1980. Control of crater morphology by gravity and target type-Mars, Earth, Moon, Lunar and Planetary Science Conference Proceedings, pp. 2159-2189.

Povilaitis, R., Robinson, M., Van der Bogert, C., Hiesinger, H., Meyer, H., Ostrach, L., 2018. Crater density differences: Exploring regional resurfacing, secondary crater populations, and crater saturation equilibrium on the moon. Planetary and Space Science 162, 41-51.

Robbins, S.J., 2018. A New Global Database of Lunar Impact Craters> 1-2 km: 1. Crater Locations and Sizes, Comparisons with Published Databases, and Global Analysis. Journal of Geophysical Research: Planets 124(4), 871-892.

Salamunićcar, G., Lončarić, S., Grumpe, A., Wöhler, C., 2014. Hybrid method for crater detection based on topography reconstruction from optical images and the new LU78287GT catalogue of Lunar impact craters. Advances in Space Research $53,1783-1797$.

Salamunićcar, G., Lončarić, S., Mazarico, E., 2012. LU60645GT and MA132843GT catalogues of Lunar and Martian impact craters developed using a Crater Shape-based interpolation crater detection algorithm for topography data. Planetary and Space Science 60, 236-247.

Senft, L.E., Stewart, S.T., 2007. Modeling impact cratering in layered surfaces. Journal of Geophysical Research: Planets 112 
Smith, D.E., Zuber, M.T., Neumann, G.A., Lemoine, F.G., Mazarico, E., Torrence, M.H., McGarry, J.F., Rowlands, D.D., Head, J.W., Duxbury, T.H., 2010. Initial observations from the lunar orbiter laser altimeter (LOLA). Geophysical Research Letters 37(18).

Wang, Y., Zhu, X., Wu, B., 2018. Automatic Detection of Individual Oil Palm Trees from UAV Images Using HOG Features and an SVM Classifier. International Journal of Remote Sensing 40(19), 7356-7370

Wang, Y., Wu, B., 2019. Active Machine Learning Approach for Crater Detection from Planetary Imagery and Digital Elevation Models. IEEE Transactions on Geoscience and Remote Sensing 57(8), 5777-5789.

Wu, B., Huang, J., Li, Y., Wang, Y., Peng, J., 2018. Rock Abundance and Crater Density in the Candidate Chang'E-5 Landing Region on the Moon. Journal of Geophysical Research: Planets 123, 3256-3272.
Wu, B., Li, F., Ye, L., Qiao, S., Huang, J., Wu, X., Zhang, H., 2014. Topographic modeling and analysis of the landing site of Chang'E-3 on the Moon. Earth and planetary science letters 405, 257-273.

Wu, B., Li, F., Hu, H., Zhao, Y., Wang, Y., Xiao, P., Li, Y., Liu, W. C., Chen, L., Ge, X., Yang, M., Xu, Y., Ye, Q., Wu, X., Zhang, H., 2020. Topographic and Geomorphological Mapping and Analysis of the Chang'E-4 Landing Site on the Far Side of the Moon. Photogrammetric Engineering \& Remote Sensing 86(4), 247-258.

Wünnemann, K., Ivanov, B., 2003. Numerical modelling of the impact crater depth-diameter dependence in an acoustically fluidized target. Planetary and Space Science 51, 831-845.

Xiao, Z., Werner, S.C., 2015. Size-frequency distribution of crater populations in equilibrium on the Moon. Journal of Geophysical Research: Planets 120, 2277-2292. 